\title{
Science yield modeling with the Exoplanet Open-Source Imaging Mission Simulator (EXOSIMS)
}

\author{
Christian Delacroix ${ }^{* a, b}$, Dmitry Savransky ${ }^{a, b}$, Daniel Garrett ${ }^{a}$, Patrick Lowrance ${ }^{c}$, Rhonda Morgan ${ }^{d}$ \\ ${ }^{a}$ Sibley School of Mechanical and Aerospace Engineering, Cornell University, Ithaca, NY 14853; \\ ${ }^{b}$ Carl Sagan Institute, Cornell University, Ithaca, NY 14853 \\ ${ }^{c}$ IPAC, California Institute of Technology, M/S 100-22, 1200 East California Blvd., Pasadena, CA \\ 91125 \\ ${ }^{d}$ Jet Propulsion Laboratory, 4800 Oak Grove Dr., Pasadena, CA 91109
}

\begin{abstract}
We report on our ongoing development of EXOSIMS and mission simulation results for WFIRST. We present the interface control and the modular structure of the software, along with corresponding prototypes and class definitions for some of the software modules. More specifically, we focus on describing the main steps of our high-fidelity mission simulator EXOSIMS, i.e., the completeness, optical system and zodiacal light modules definition, the target list module filtering, and the creation of a planet population within our simulated universe module. For the latter, we introduce the integration of a recent mass-radius model from the FORECASTER software. We also provide custom modules dedicated to WFIRST using both the Hybrid Lyot Coronagraph (HLC) and the Shaped Pupil Coronagraph (SPC) for detection and characterization, respectively. In that context, we show and discuss the results of some preliminary WFIRST simulations, focusing on comparing different methods of integration time calculation, through ensembles (large numbers) of survey simulations.
\end{abstract}

Keywords: high contrast imaging, exoplanets, space missions, WFIRST, coronagraphs, end-to-end simulator, integration time, zodiacal light

\section{INTRODUCTION}

In addition to allowing for new detections of exoplanets and exozodiacal disks, direct imaging also enables astrometry and photometry of these objects, and allows for better estimation of exoplanet orbital parameters (e.g., $\beta$ Pictoris, ${ }^{1} \mathrm{HR}$ $8799^{2,3}$ ). Furthermore, high-resolution spectroscopic analysis can ultimately lead to determining the atmospheric structure and chemical composition of exoplanets. ${ }^{4}$ Direct imaging is therefore highly desirable, but also challenging. It requires combining technologies such as coronagraphy, wavefront sensing and control, pointing jitter control, and software solutions leading to post-processing gains in terms of contrast. So far, only a few dozen large bright planets have been imaged, mostly around young and nearby stars, and on fairly long-period orbits. In order to extend this parameter space, astronomers will most likely need to send out their instrumentation on spacecraft, and observe exoplanets directly from space. In this context, the WFIRST (Wide-Field Infrared Survey Telescope) NASA mission will be equipped with coronagraphs ${ }^{5}$ to directly image and spectrally characterize exoplanets and exozodiacal disks around nearby stars.

As part of the WFIRST Preparatory Science investigation, we are developing the Exoplanet Open-Source Imaging Mission Simulator (EXOSIMS) ${ }^{6}$ designed to allow for systematic exploration of expected science yields over the course of a specific mission. A schematic depiction of the EXOSIMS modular architecture is given in Fig. 1. This modular structure allows users to investigate multiple missions or system designs by only modifying individual modules without having to redefine the unaffected ones. The instantiation of all modules begins with the construction of a MissionSimulation object, after loading the input specification from a single script file (see Fig. 1, green box). All other module objects are then instantiated, with the arrows indicating calls to object constructors in the order shown in the flow

*cd458@cornell.edu; sioslab.mae.cornell.edu

Modeling, Systems Engineering, and Project Management for Astronomy VII, edited by George Z. Angeli, Philippe Dierickx, Proc. of SPIE Vol. 9911, 991119

(c) 2016 SPIE $\cdot$ CCC code: $0277-786 X / 16 / \$ 18 \cdot$ doi: $10.1117 / 12.2233913$

Proc. of SPIE Vol. $9911991119-1$ 


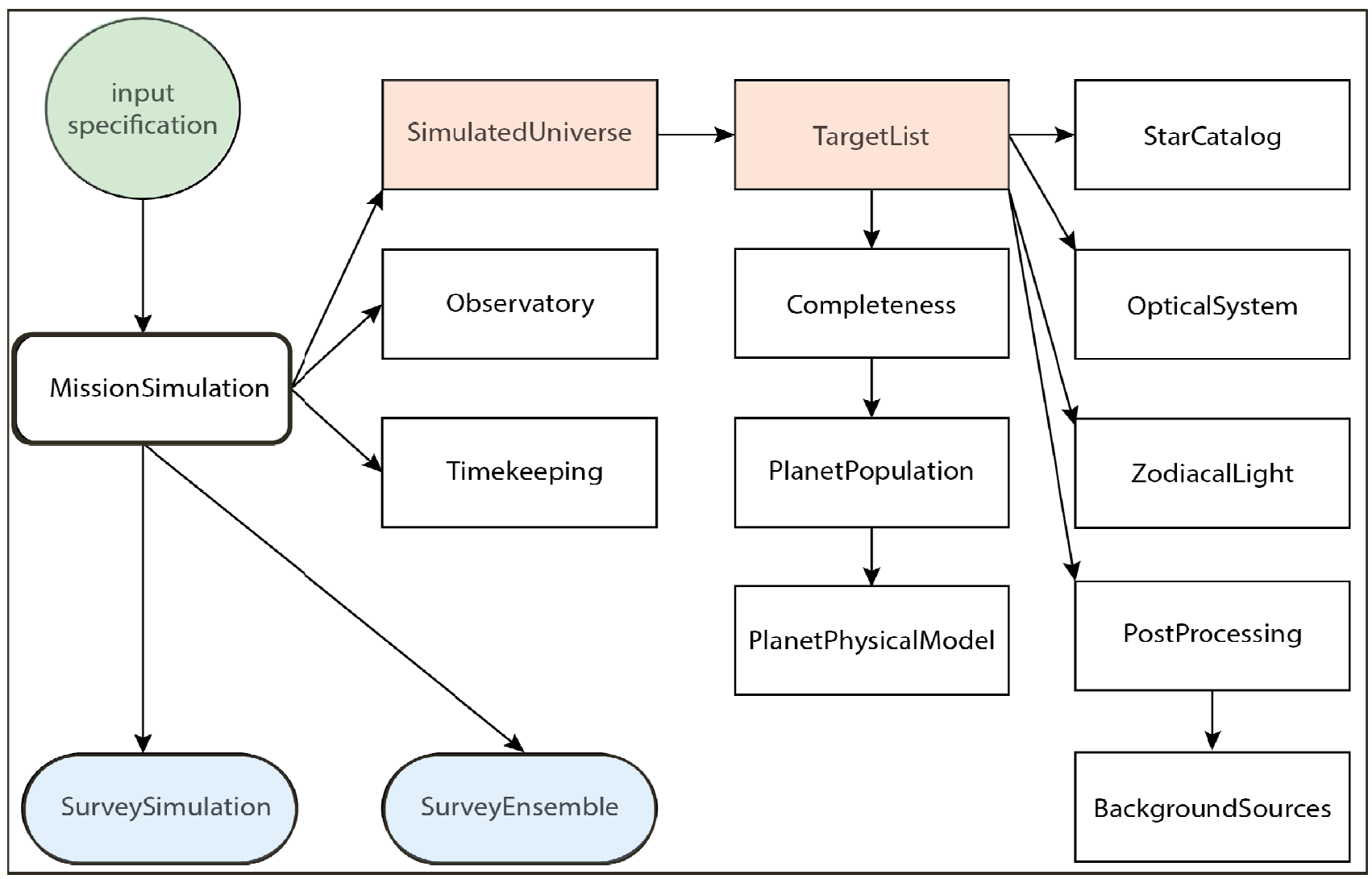

Figure 1. Flow chart describing the instantiation of an end-to-end simulation. Each software module is represented by a box, and corresponds to a simulation step. The input specification (green box) is a single script file loaded by the main module MissionSimulation. The code operates by successive interactions between modules, as indicated by the arrows. The TargetList and SimulatedUniverse modules (red boxes) are being described in Sect. 2 and Sect. 3, respectively. The SurveySimulation and SurveyEnsemble modules (blue boxes) are responsible for running either one or a large number of simulations, respectively.

chart. All module references are passed to the top calling module, such that the MissionSimulation object has direct access to all other modules as its attributes. The last two constructed modules (see Fig. 1, blue boxes) are responsible for performing either one (SurveySimulation) or a large number (SurveyEnsemble) of simulations based on all of the input parameters and models. Each simulation returns the mission timeline, i.e., an ordered list of simulated observations of various targets on the target list along with their outcomes.

In this paper, we give an update on our ongoing development of EXOSIMS. More specifically, we focus on our upgrades to two core steps of the software (see Fig. 1, red boxes) - the creation of a target list and the simulation of the whole universe, i.e., the planets around the target stars. In Sect. 2, we detail some of the main modules involved in constraining the mission simulation target list, namely the Completeness module, the OpticalSystem module, and the ZodiacalLight module. In Sect. 3, we introduce the integration of a recent probabilistic mass-radius relation, made available through the FORECASTER software. ${ }^{7}$ Finally, in Sect. 4, we show results of ensembles of survey simulations, with a comparison of different methods of calculating the integration times, that have been integrated to the OpticalSystem module. ${ }^{8-10}$

\section{TARGET LIST FILTERING}

The TargetList module collects information from eight downstream modules: StarCatalog, OpticalSystem, ZodiacalLight, PostProcessing, BackgroundSources, Completeness, PlanetPopulation, and PlanetPhysicalModel. The target list can contain pre-determined target stars where planets are known to exist from previous surveys (e.g., radial- 
velocity surveys, transit surveys). Alternatively, the target list can contain all of the targets from a star catalog where a planet with specified parameter ranges could be observed. In that particular case, the TargetList module first copies the whole catalog (e.g. SIMBAD) by running the StarCatalog module and removes stars with any NaN attributes, to populate the target list with usable attribute values, with units defined as Python Astropy Quantities. Then, TargetList performs a population filtering based on selected criteria. For instance, the prototype implementation can do the following:

- remove binary stars;

- remove systems not meeting the single-visit completeness threshold;

- $\quad$ remove systems with planets outside of the coronagraph working angles;

- remove systems where integration time is longer than maximum integration time.

The following subsections illustrate the definition of some of the main parameters required to constrain the target list, i.e. the completeness value, the optical system throughput, contrast, inner working angle (IWA) and outer working angle (OWA), the zodiacal light, and the integration time.

\subsection{Completeness module}

We first calculate a joint probability density function (PDF) of the planet population's apparent separation (s) together with the difference in brightness between the star and the planet $(\Delta \mathrm{mag})$. This photometric restriction on exoplanet observability is introduced by the telescope optics. ${ }^{11}$ Then, we evaluate a completeness level for each target. The completeness is the $2 \mathrm{D}$ integration of a portion (i.e., the cumulative density function) of a specific PDF such as the one shown in Fig. 2. The integrated portion is defined for a specific target star, and a specific imaging instrument, with given IWA, OWA, and a $\Delta$ mag limiting value. The calculated value corresponds to the probability that a particular observatory, while observing a star for the first time, will detect a planet belonging to the assumed population. These completeness values are then updated throughout the mission, for target stars that were previously observed. An exact formulation of the multivariate integral representing such a completeness joint probability density function was recently derived in Ref. 12.

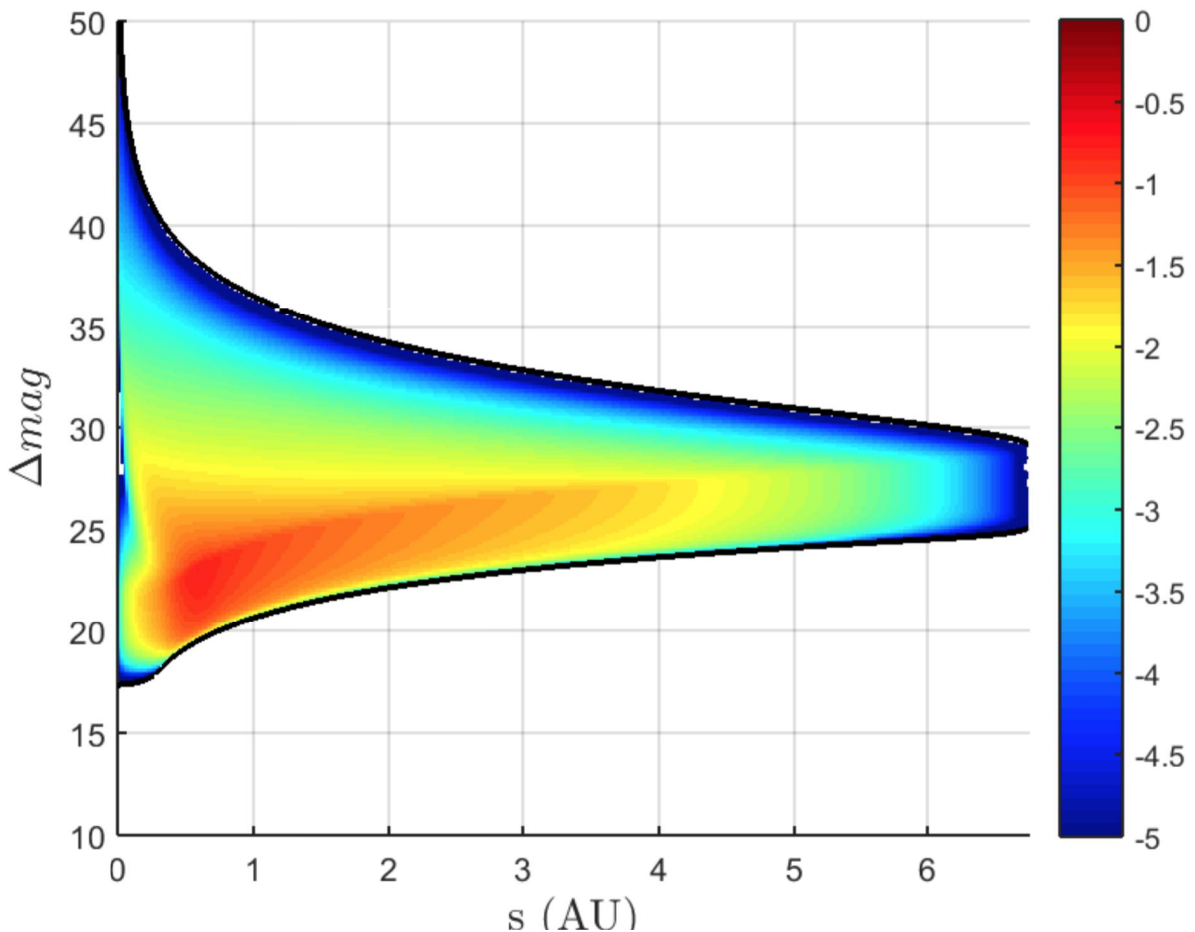

Figure 2. Example of joint probability density function. The color is log-scaled (base 10) of the probability density in units of $\mathrm{AU}^{\wedge}-1 \mathrm{mag}^{\wedge}-1$. The black lines represent minimum and maximum values for which the completeness joint probability density function is nonzero. From Ref. 12. 


\subsection{ZodiacalLight module}

The local zodiacal light surface brightness is usually ${ }^{11,8}$ given by a uniform brightness of 23 mag $\operatorname{arcsec}^{\wedge}-2$. However, zodiacal brightness changes for each observation direction, and at each wavelength. The ZodiacalLight module of EXOSIMS integrates a coordinate and wavelength dependence for each target. As in Ref. 8, this dependence is obtained by interpolating Tables 17 and 19 of diffuse night sky brightness presented in Ref. 13. This interpolation preliminarily requires coordinate transformations. In fact, the tables give zodiacal light in terms of geocentric ecliptic coordinates $\lambda-\lambda_{\odot}, \beta$ with the zero point of $\lambda$ in the Sun, as illustrated in Fig. 3. The target coordinates, as copied from the star catalog, are defined as right ascension $(\alpha)$ and declination $(\delta)$ in the International Celestial Reference System (ICRS), with its origin at the barycenter of the Solar System. These ICRS $\alpha, \delta$ coordinates must be transformed into observatorycentered ecliptic coordinates $\lambda, \beta$ with respect to the spacecraft: $\overline{O T}=\overline{S T}-\overline{S O}$.

Then, Leinert's ecliptic coordinates $\lambda-\lambda_{\odot}, \beta$ can be easily obtained by subtracting the longitude of the Sun with respect to the observatory $\left(\lambda_{\odot}\right)$. The latter is simply the anti-supplementary angle of the longitude of the observatory with respect to the Sun: $\lambda_{\odot}=\lambda_{\text {obs }}+180^{\circ}$.

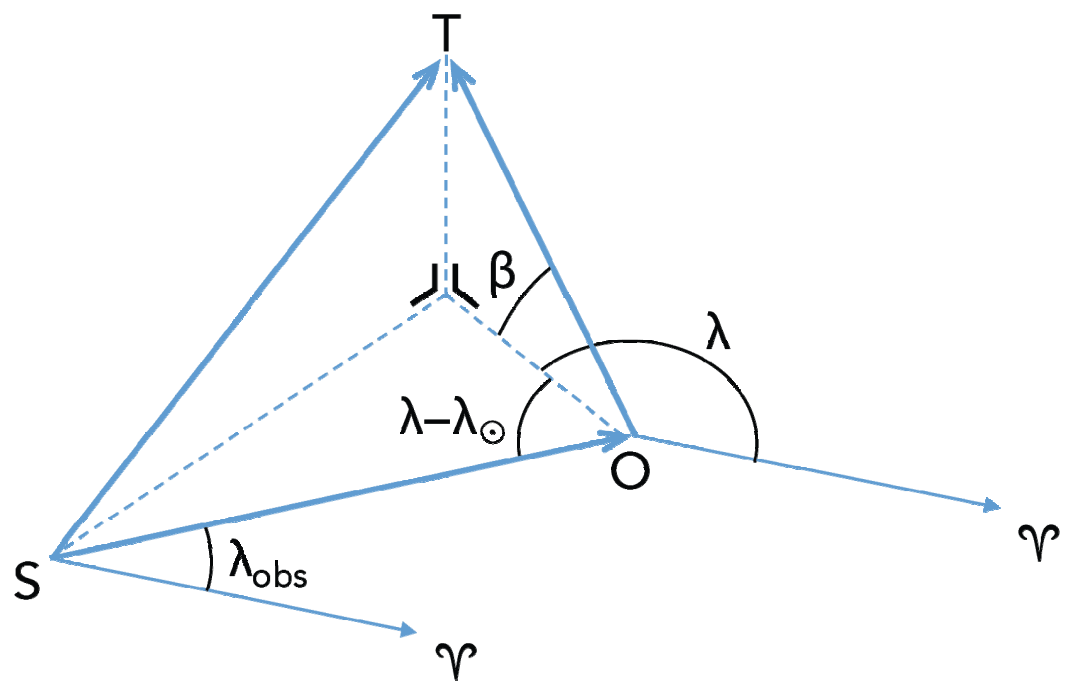

Figure 3. Diagram illustrating the target coordinates definition, $S$ being the position of the sun, $O$ the observatory, and $T$ the target. $\checkmark$ represents the direction of the vernal equinox. The brightness of the local zodiacal light, as seen from the observatory, is given by Leinert's table, ${ }^{13}$ for the ecliptic longitudes and latitudes $\left(\lambda-\lambda_{\odot}, \beta\right)$ with the zero point of longitude in the direction of the Sun. The longitude of the Sun with respect to the observatory $\left(\lambda_{\odot}\right)$ and the longitude of the observatory with respect to the Sun $\left(\lambda_{\text {obs }}\right)$ are anti-supplementary angles.

\subsection{OpticalSystem module}

The Optical System module contains all of the necessary information to describe the effects of the telescope and starlight suppression system on the target star and planet wavefronts. For instance, the user can specify angular separation and wavelength dependent contrast and throughput definitions, together with Point Spread Functions (PSF) for on- and offaxis sources. ${ }^{14,15}$ Because these parameters are wavelength dependent, they are defined as callable functions. As an example, Fig. 4 (left) shows the performance curves of a coronagraph at the specific wavelength value of $550 \mathrm{~nm}$. Both the contrast and throughput input data are angular separation-dependent arrays, where the array first column contains the separations in arcsec. These curves allow for the extraction of specific parameters including the instrument IWA and OWA defined as the min and max angular separations at 50\% normalized throughput, as well as the average and maximum contrast and throughput over the IWA-OWA range. Fig. 4 (right) shows the on- and off-axis normalized PSFs for the same coronagraph. Both horizontal sequences of PSFs correspond to the same data, but with two different colorbar scales: the upper sequence is logarithmic, whereas the lower sequence is linear. Each PSF is observed at a specific off-axis separation, in units of $\lambda / \mathrm{D}, \lambda$ being the instrument central wavelength and $\mathrm{D}$ the telescope primary mirror diameter. The white circles on the upper sequence correspond to the core of the PSFs, with a FWHM circle 
diameter. Based on this PSF sequence, one can easily derive the original contrast and throughput curves by performing simple aperture photometry. As a visual example, the normalized throughput at a separation of $\sim 3 \lambda / \mathrm{D}(\sim 0.14 \mathrm{arcsec})$ is about $50 \%$, which matches the value of the IWA defined by the throughput curve.
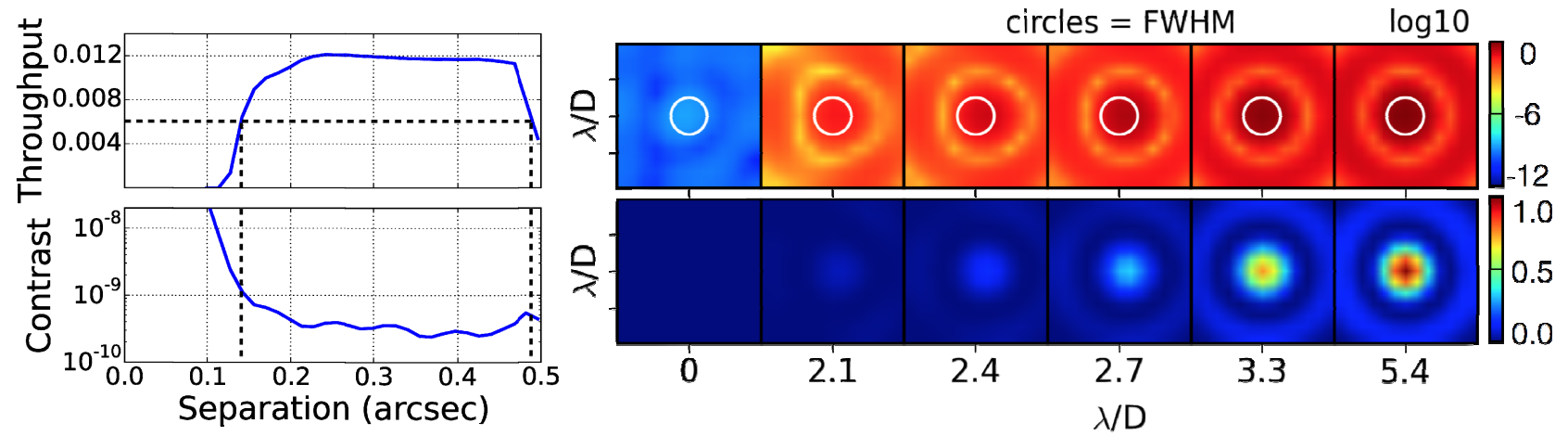

Figure 4. Example of a starlight suppression system performance, given as input to EXOSIMS. Left: the throughput and the contrast curves, as functions of the angular separation, for a specific wavelength value. The instrument IWA and OWA are calculated at 50\% throughput. Right: an off-axis PSF sequence represented both on a logarithmic and a linear scale, for comparison. Aperture photometry is performed by integrating the FWHM white circles, to reproduce the throughput and contrast curves.

\subsection{Integration time calculation}

As previously stated, the TargetList module filters out systems with integration times larger than an integration cutoff value set by the user. By doing so, the software ensures that no observation will start with an unacceptably long integration time, reducing severely the remaining life time of the mission. The OpticalSystem prototype module calculates the electron count rates for the planet signal $C_{p}(\lambda)$ and the background noise $C_{b}(\lambda)$ as functions of the wavelength $\lambda$, based on the spectral flux density electron count rate $C_{\mathcal{F} 0}(\lambda)$

$$
C_{\mathcal{F} 0}(\lambda)=\epsilon A \Delta \lambda T(\lambda) \mathcal{F}_{0}(\lambda)
$$

where $\epsilon$ is the quantum efficiency, $A$ is the pupil area, $\Delta \lambda$ is the bandwidth, $T(\lambda)$ is the total optical system throughput, and $\mathcal{F}_{0}(\lambda)$ is the spectral flux density function. The latter is obtained by using the following empirical expression ${ }^{16,17}$

$$
\mathcal{F}_{0}(\lambda)=10^{\left(4.01-\frac{\lambda-550}{770}\right)}
$$

which returns $\sim 10^{4}$ photon $\mathrm{s}^{\wedge}-1 \mathrm{~cm}^{\wedge}-2 \mathrm{~nm}^{\wedge}-1$ at the specific wavelength value of $550 \mathrm{~nm}$. The planet signal electron count is then given by

$$
C_{p}(\lambda)=C_{\mathcal{F} 0}(\lambda) 10^{-0.4(\mathrm{Vmag}+\Delta \mathrm{mag})}
$$

and the background noise electron count is given by ${ }^{10}$

$$
C_{b}(\lambda)=\operatorname{ENF}^{2}\left(C_{s r}(\lambda)+C_{z l}(\lambda)+C_{d c}+C_{c c}\right)+C_{r n}
$$

with the following definitions:

- $\quad$ excess noise factor ENF $=\sqrt{2}(\mathrm{EM}-\mathrm{CCD})$ or $1(\mathrm{CCD})$;

- $\quad$ suppressed starlight residuals $C_{s r}(\lambda)=C_{\mathcal{F} 0}(\lambda) 10^{-0.4 V \mathrm{mag}} Q(\lambda)$ with $Q(\lambda)=$ coron. contrast;

- $\quad$ zodiacal light (local + exozodi) $C_{z l}(\lambda)$;

- $\quad$ dark current $C_{d c}$;

- $\quad$ clock-induced-charge $C_{c c}$;

- readout noise $C_{r n}$. 
The total noise budget is illustrated in Fig. 5. Four virtual planet PSFs are being imaged at various angular separations from their host star, ranging from 2 to $7 \lambda / \mathrm{D}$. The sequence of images shows that the background noise consists primarily of the residual diffracted starlight speckles, due to wavefront errors. Without any image post-processing, the scattered light dominates the background variance and the planet signals cannot be retrieved. A 10-time post-processing gain already reduces significantly the speckle noise, and allows the detection of two or three of the candidates.

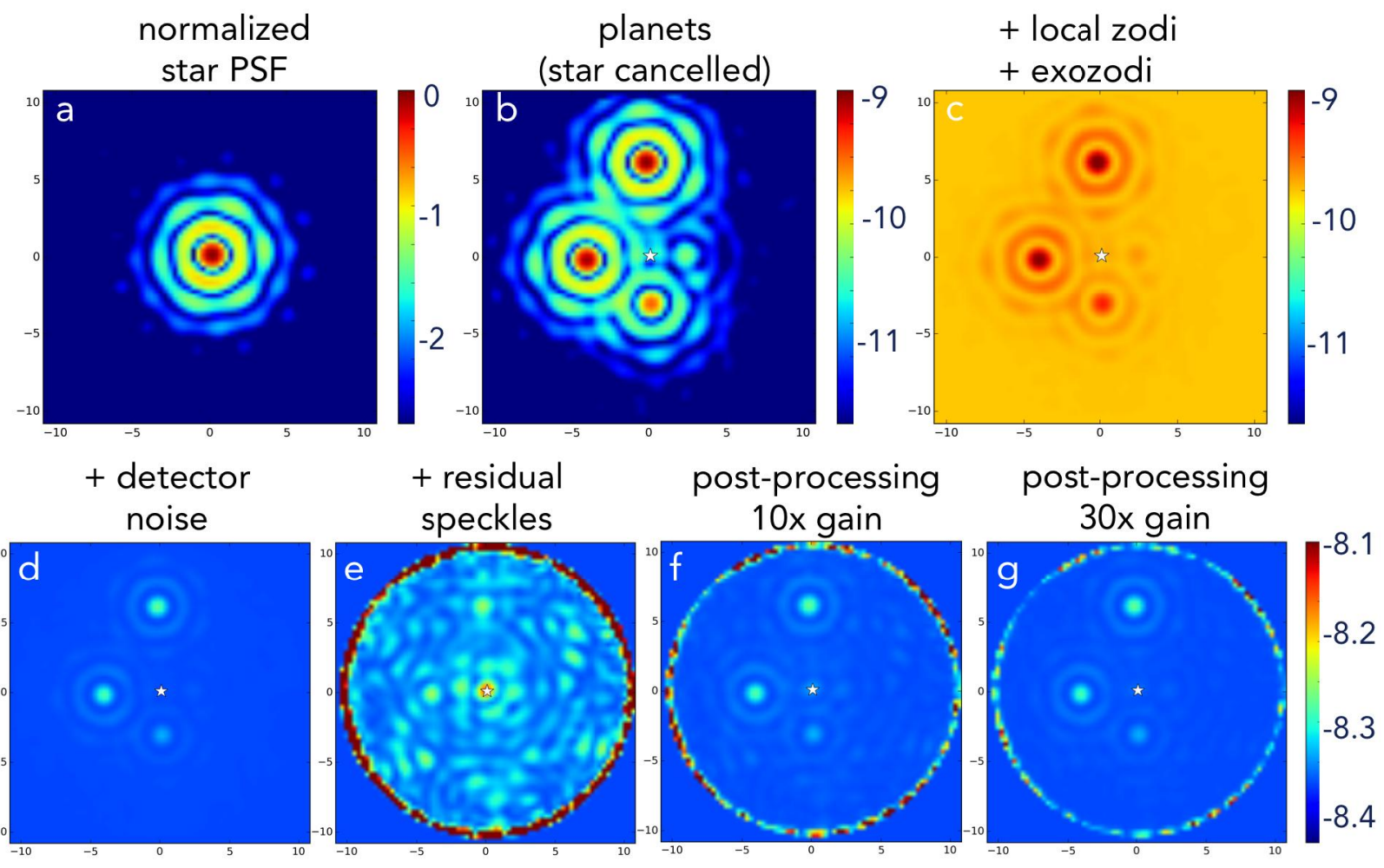

Figure 5. Sequence of simulated images of a target with four virtual companion planets. Image $a$ is the target normalized PSF, with a logarithmic colorbar scale. Image $b$ assumes a perfect star cancellation, and reveals clearly the four planet PSFs at various angular separations, with the addition of the diffuse source of total zodiacal light in image $c$. Images $d$ and $e$ include the detector noise (dark current, clock-induced charge, readout) and the diffracted starlight residual speckles. Images $f$ and $g$ are two examples of post-processing gain, allowing to retrieve two or three of the planets that were previously hidden by speckle noise.

\section{MASS-RADIUS RELATION USING FORECASTER}

After populating the target list and filtering based on selected criteria, the SimulatedUniverse module creates a synthetic universe by populating planetary systems about some or all of the stars in the target list. Each planetary system is generated based on the statistics encoded in the PlanetPopulation module, so that the overall planet occurrence and multiplicity rates are consistent with the provided distribution functions. The PlanetPopulation module encodes the density functions of all required planetary parameters, including semi-major axis, eccentricity, orbital orientation, radius, mass, and geometric albedo. Certain parameter models may be empirically derived while others may come from analyses of observational surveys, and calculated via the PlanetPhysicalModel module. Most planet indirect detections (radial velocity, microlensing or transit) can measure either the planet mass or its radius, but not both. Future missions will need to forecast the missing quantity (M or R) by means of an MR relation, in order to predict detectability. At the first level, detectability of exo-atmosphere is proportional to $R^{2} / M$. Planets with known radius and mass will be easier to characterize with follow-up observations such as JWST, ${ }^{18}$ which will not have time for both measures given the limited supply of cryogen onboard. 
In practice, transit missions (e.g. TESS ${ }^{19}$ ) need the forecast of the mass based upon the radius, while radial-velocity (RV) missions need the radius based upon the mass. Fig. 6 illustrates the whole known RV planets universe generated by the PlanetPopulation module. For a large majority of these planets, only the mass is available and the radius needs to be calculated. To do so, we use a recent probabilistic MR relation model called FORECASTER, ${ }^{7}$ that we integrated to EXOSIMS in a dedicated PlanetPhysicalModel module. In addition to forecasting, the inference of the MR relation enables classification, which has significant impact in some cases, e.g., the occurrence rate of exo-Earths $\eta_{\oplus}$ increases by $72 \%$ when altering their definition from $R<1.5 R_{\oplus}$ to $R<2.0 R_{\oplus} \cdot{ }^{20}$ The classification of FORECASTER leads to four categories of worlds (Terran, Neptunian, Jovian, Stellar) and three transitions. The Neptunian-Jovian transition is not as physically intuitive as the other two. A plausible physical interpretation is that a Neptunian radius grows rapidly as more mass is added, whereas a Jovian mass is sufficient for gravitational self-compression to start reversing the growth. ${ }^{7}$

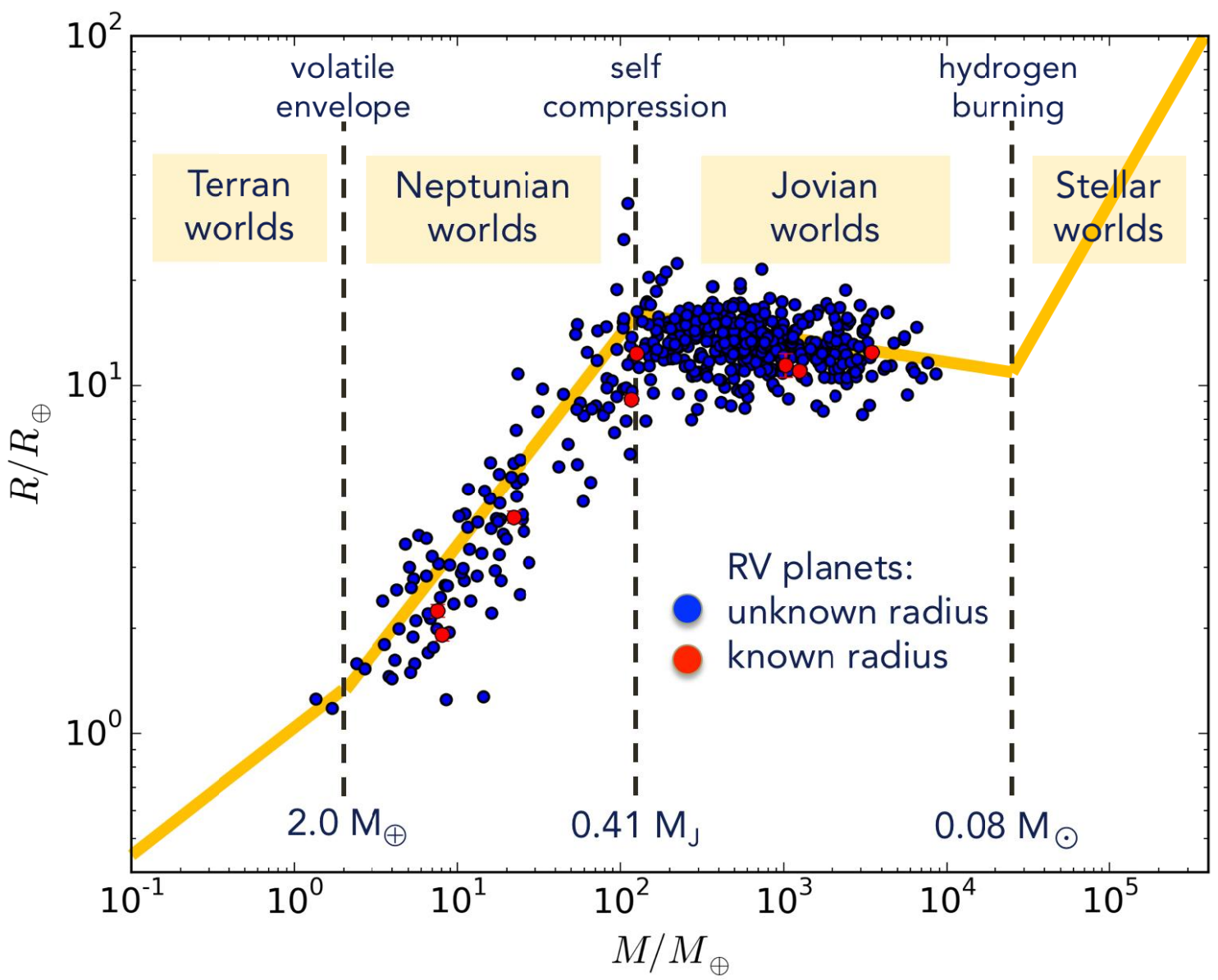

Figure 6. Radius and mass values for the whole planet population, generated from known radial-velocity planets. Only a few of these planets (red dots) have both their mass and radius measured. The large majority (blue dots) have their radius value provided by the MR relation from FORECASTER. Note the classification in four worlds: Terran worlds (including dwarf planets), Neptunian worlds, Jovian worlds (including brown dwarfs), and Stellar worlds (K, M, and late-type G dwarf stars). Figure based on Ref. 7.

\section{SURVEY ENSEMBLE RESULTS}

After the execution of the TargetList and the SimulatedUniverse modules, two additional modules are called (TimeKeeping and the Observatory) to predict which target stars are observable at a specific time during the mission simulation and which are unobservable due to bright objects within the field of view, such as the sun, moon, and solar 
system planets. The MissionSimulation is then ready to start an observing survey of the selected targets and their planet companions. For each observation, an integration time is calculated. The integration time of a specific planet, with a specific science instrument, involves the wavelength-dependent expressions (3) and (4) introduced in Sect. 2.4. These equations are used to obtain both the planet signal and the background noise electron counts, $C_{p}(\lambda)$ and $C_{b}(\lambda)$. Different methods exist to determine the integration times. Here, we focus on a comparison study of two methods, described in the following papers:

- Kasdin\&Braems $2006^{9}$ : The authors define a false alarm probability $P_{\mathrm{FA}}=3 \times 10^{-5}$ and a missed detection probability $P_{\mathrm{MD}}=10^{-3}$. The integration time is given by

$$
t(\lambda)=\frac{C_{b}(\lambda)}{C_{p}(\lambda)^{2} \overline{\Delta \alpha}}\left(\left(1-P_{\mathrm{FA}}\right)-\left(1-P_{\mathrm{MD}}\right) \sqrt{1+C_{p} \sum \overline{\mathrm{PSF}}_{\mathrm{ij}}^{3} / C_{b} \sum \overline{\mathrm{PSF}}_{\mathrm{ij}}^{2}}\right)^{2}
$$

where $\overline{\Delta \alpha}$ is the dimensionless pixel size, and $\overline{\mathrm{PSF}}$ is the normalized PSF.

- Nemati $2014^{10}$ : The author defines a signal-to-noise ratio level (e.g. SNR = 5). The integration time is then given by

$$
t(\lambda)=\frac{C_{b}(\lambda) \mathrm{SNR}^{2}}{C_{p}(\lambda)^{2}-\left(\operatorname{SNR} f_{\mathrm{PP}} C_{s r}(\lambda)\right)^{2}}
$$

where $f_{\mathrm{PP}}$ is the expected gain due to post-processing.

The results of our comparison study are illustrated in Fig. 7 and are obtained with data produced from a small but sufficient ensemble $(\mathrm{N} \sim 500)$ of survey simulations. Larger ensembles $(\sim 5000)$ will be used for final mission yield evaluations. The baseline implementation of the SurveyEnsemble module is a simple looping function that executes the desired number of simulations sequentially, and can be run in a limited number of separate IPython terminals for parallel computing. A specific module is also being developed, implementing a locally parallelized looping based on IPython Parallel. ${ }^{21}$ The results shown in Fig. 7 indicate a similar yield of unique detections with both methods, and a slightly increased yield of total detections with the Kasdin\&Braems method $\left(P_{\mathrm{FA}}=3 \times 10^{-5}\right.$ and $\left.P_{\mathrm{MD}}=10^{-3}\right)$ compared to the Nemati method $(\mathrm{SNR}=5)$. The former appears to be somewhat more optimistic, producing shorter integration times.
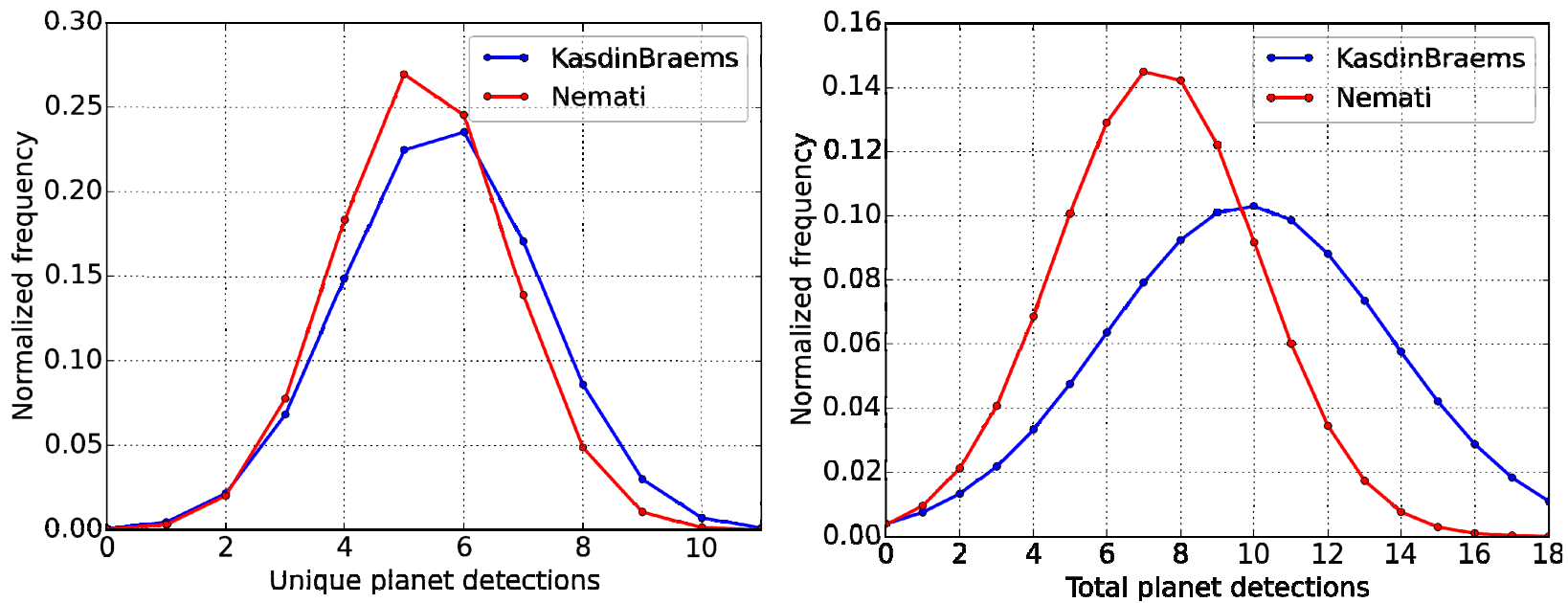

Figure 7. Probability density function of unique planet detections (left-hand) and total planet detections (right-hand). The plotted curves correspond to the Kasdin\&Braems method (Eq. 5, blue curves) and Nemati method (Eq. 6, red curves). 


\section{CONCLUSIONS}

We provide an update on the ongoing development of EXOSIMS. We give a detailed description of the TargetList module filtering, based on selected criteria introduced by the user, including completeness threshold, optical system working angles, and integration time cutoff. The completeness joint probability recently derived by our team is presented briefly. We also show results of wavelength dependent contrast and throughput curves obtained from a sequence of onand off-axis source PSFs, using aperture photometry on the PSF core region delimited by the FWHM. The working angle range (IWA-OWA) is then determined by the OpticalSystem module based on the calculated throughput curve. The prototype OpticalSystem module is now also responsible for the planet signal and background noise electron count. In that context, we study different existing methods for calculating the integration time, and we provide specific OpticalSystem modules for each method. In addition, we explore new planet property models, and we integrate the MR relation model from the FORCASTER software, to predict the missing property (mass or radius) of previously detected planets (transits, radial velocities). Finally, we analyze simulation ensembles data, focusing on the comparison between the newly implemented integration time calculation methods. Our preliminary results show consistent unique detection yields, with slightly different mean integration times. Further analysis is ongoing. EXOSIMS will continue to be further developed through the final release in May of 2017, and will be used to continuously refine the modeling of the WFIRST coronagraph science.

\section{ACKNOWLEDGEMENT}

This work is supported by NASA Grant Nos. NNX14AD99G (GSFC) and NNX15AJ67G (WPS).

\section{REFERENCES}

[1] Lagrange, A.-M., Gratadour, D., Chauvin, G., et al., "A probable giant planet imaged in the $\beta$ Pictoris disk. VLT/NaCo deep L'-band imaging," $A \& A$ 493, L21-L25 (2009).

[2] Marois, C., Macintosh, B., Barman, T., et al., "Direct Imaging of Multiple Planets Orbiting the Star HR 8799," Science 322, 1348-1352 (2008).

[3] Marois, C., Zuckerman, B., Konopacky, Q. M. et al., "Images of a fourth planet orbiting HR 8799," Nature 468, 1080-1083 (2010).

[4] Konopacky, Q., Barman, T., Macintosh, B., and Marois, C., "Detection of Carbon Monoxide and Water Absorption Lines in an Exoplanet Atmosphere," Science 339, 1398-1401 (2013).

[5] Noecker, M., Zhao, F., Demers, R., et al., "Coronagraph instrument for WFIRST-AFTA," JATIS 2(1), 011001 (2016).

[6] Savransky, D., and Garrett, D., "WFIRST-AFTA Coronagraph Science Yield Modeling with EXOSIMS," JATIS 2(1), 011006 (2016).

[7] Chen, J., and Kipping, D., "Probabilistic Forecasting of the Masses and Radii of Other Worlds," arXiv: 1603.08614 (2016).

[8] Stark, C., Roberge, A., Mandell, A., and Robinson, T., "Maximizing the ExoEarth Candidate Yield from a Future Direct Imaging Mission," ApJ 795, 122 (2014).

[9] Kasdin, J., and Braems, I., "Linear and Bayesian Planet Detection Algorithms for the Terrestrial Planet Finder," ApJ 646, 1260-1274 (2006).

[10] Nemati, B., "Detector Selection for the WFIRST-AFTA Coronagraph Integral Field Spectrograph," Proc. SPIE 9143, 91430Q (2014).

[11] Brown, R., "Single-Visit Photometric and Obscurational Completeness," ApJ 624, 1010 (2005).

[12] Garrett, D., and Savransky, D., "Analytical Formulation of the Single-Visit Completeness Joint Probability Density Function," accepted to $A p J(2016)$.

[13] Leinert, C., Bowyer, S., Haikala, L., et al., "The 1997 reference of diffuse night sky brightness," A\&AS 127, 199 (1998).

[14] Krist, J., Nemati, B., and Mennesson, B., "Numerical modeling of the proposed WFIRST-AFTA coronagraphs and their predicted performances," JATIS 2(1), 011003 (2016). 
[15] Trauger, J., Moody, D., Krist, J., and Gordon, B., "Hybrid Lyot coronagraph for WFIRST-AFTA: coronagraph design and performance metrics," JATIS 2(1), 011013 (2016).

[16] Pecaut, M., and Mamajek, E., "Intrinsic colors, temperatures, and bolometric corrections of pre-main-sequence stars," ApJS, 208, 22pp (2013).

[17] Traub, W., Breckinridge, J., Greene, T., et al., "Science yield estimate with the WFIRST-AFTA coronagraph," JATIS 2(1) (2016).

[18] Seager, S., Deming, D., and Valenti, J., "Transiting Exoplanets with JWST," Proc. Astrophysics and Space Science, 10, 123-145 (2009).

[19] Ricker, G., Winn, J., Vanderspek, R., et al., "Transiting Exoplanet Survey Satellite (TESS)," Proc. SPIE 9143, 914320 (2014).

[20] Foreman-Mackey, D., Hogg, D., and Morton, T., "Exoplanet population inference and the abundance of Earth analogs from noisy, incomplete catalogs," ApJ 795, 64 (2014).

[21] Perez, F., and Granger, B., "IPython: a system for interactive scientific computing," Comput. Sci. Eng. 9(3), 2129 (2007). 